This item was submitted to Loughborough's Institutional Repository (https://dspace.lboro.ac.uk/) by the author and is made available under the following Creative Commons Licence conditions.

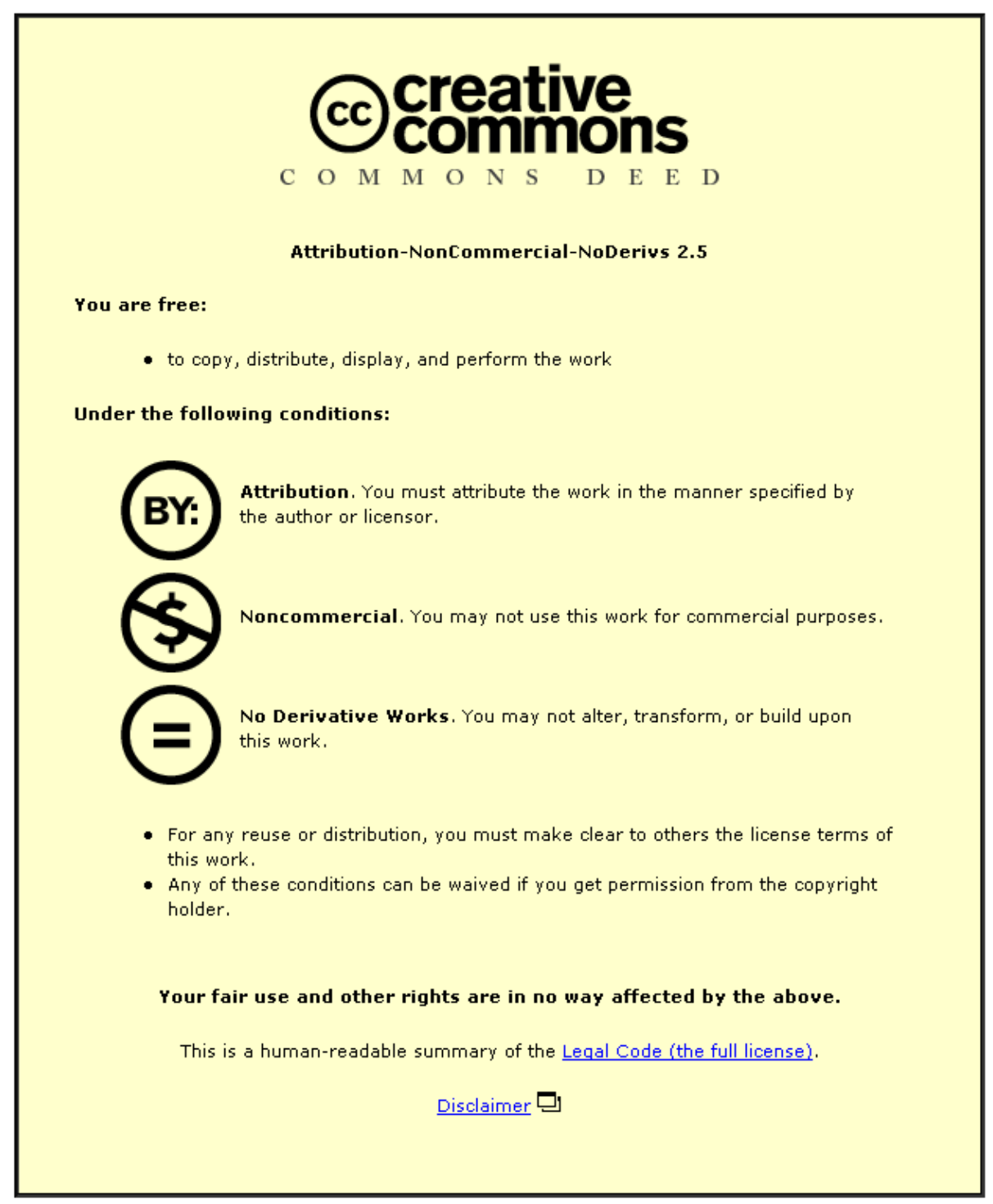

For the full text of this licence, please go to: http://creativecommons.org/licenses/by-nc-nd/2.5/ 


\title{
Characterising the Linearity of an Optically Controlled Photoconductive Microwave Switch
}

\author{
Emma K. Kowalczuk ${ }^{1}$, Chinthana J. Panagamuwa ${ }^{2}$, Rob D. Seager ${ }^{3}$, J. (Yiannis) C. Vardaxoglou ${ }^{4}$ \\ Wireless Communications Group, Electronic and Electrical Engineering, Loughborough University, \\ Loughborough, Leicestershire, LE11 3TU, U.K \\ ${ }^{1}$ E.K.Kowalczuk@lboro.ac.uk \\ ${ }^{2} \mathrm{C} . J \cdot$.Panagamuwa@lboro.ac.uk \\ ${ }^{3}$ R.D.Seager@lboro.ac.uk \\ ${ }^{4}$ J.C.Vardaxoglou@lboro.ac.uk
}

\begin{abstract}
The linearity response of a photoconductive switch on microstrip line is presented at $2 \mathrm{GHz}$. A silicon switch is exposed to incident signal power of up to $1 \mathrm{~W}$ and controlled via illumination with a range of optical intensities at a wavelength of $980 \mathrm{~nm}$ in order to characterise the linearity in terms of harmonic content. Reported single tone output third order intercept (TOI) was measured as $63 \mathrm{dBm}$ under $200 \mathrm{~mW}$ of optical incident light. The study presents photoconductive switches as a promising alternative to conventional microwave switches in high power applications.
\end{abstract}

\section{INTRODUCTION}

There is growing demand in the wireless communications industry for high power, low loss microwave switches, particularly for the purpose of reconfiguring circuitry or antenna geometry to extend functionality in terms of frequency, polarization and directivity and also to reduce system size. A number of mechanisms allow adaptivity of circuit elements, including PIN and varactor diodes and MEMS (Micro Electro-Mechanical Systems) switches. The downside to these approaches is that they are controlled by electrical bias lines which may affect the behaviour of the RF signal. Diodes are also notoriously non linear, potentially compromising performance, whereas MEMS have limited power handling. As an alternative, photoconductive microwave switches are available with the advantage of control via a light source, allowing perfect thermal and electromagnetic isolation between the controlling optical beam and the controlled RF signal, which is ideal for antenna applications.

For wireless applications which require high power RF signals, linearity becomes an important device characteristic. Linearity dictates the independence of device impedance from RF input signal power, and is typically monitored through second and third order harmonics. Linearity of photoconductive switches has not been widely reported beyond $1 \mathrm{~W}$ of incident RF power [1], and not been reported for an optically controlled switch made from a single semiconductor material. This study will report on the linearity of a photoconductive switch when exposed to $1 \mathrm{~W}$ of incident RF power and the effect that light intensity of the controlling light source has on linearity.

A review of alternative microwave switches' linearity is presented along with experimental setup and linearity measurements including single tone second and third order intercept points and switch isolation for an optically controlled silicon photoconductive switch at $2 \mathrm{GHz}$. The study builds towards future work which will report the upper linearity limit of the photoconductive switch through establishing the $1 \mathrm{~dB}$ compression point, verifying its excellent high power handling of RF signals.

\section{REVIEW OF SWITCH LINEARITY}

Microwave switches consisting of PIN and varactor diodes, MEMS switches and photoconductive switches are widely being researched to reconfigure wireless communications systems such as antennas [2] and circuitry including filters, amplifier matching circuits and couplers [3]. Since linearity of such systems is often limited by the switching device, there is continuous research into improving microwave switch linearity in reconfigurable circuits; a summary of a selection is presented in Table 1 in terms of Third Order Intercept (TOI), 3rd Order Input Intermodulation Product (IIP3) and Output IP3 (OIP3). To clarify, the measurement of IIP3 relates specifically to the intercept point for a two tone intermodulated signal with reference to the input power. The OIP3 value refers to the intercept point for a two tone signal with reference to the output power, whereas a TOI result in this study corresponds to a single tone measurement of intercept point referenced to the switch's output power.

TABLE I

CHARACTERISTICS OF HETEROSTRUCTURE PHOTOCONDUCTIVE SWITCH AND MICROWAVE SWITCHES USED IN RECONFIGURABLE APPLICATIONS

\begin{tabular}{|l|l|l|l|l|}
\hline & $\begin{array}{l}\text { Frequency } \\
(\mathbf{G H z})\end{array}$ & $\begin{array}{l}\text { Isolation } \\
(\mathbf{d B})\end{array}$ & $\begin{array}{l}\text { Power } \\
(\mathbf{W})\end{array}$ & $\begin{array}{l}\text { Linearity } \\
(\mathbf{d B m})\end{array}$ \\
\hline $\begin{array}{l}\text { Heterostructure } \\
\text { PCS, single [1] }\end{array}$ & 0.96 & 25 & $>1$ & TOI 65 \\
\hline $\begin{array}{l}\text { Single PIN diode } \\
{[5]}\end{array}$ & 5.6 & 20 & $>3$ & IIP3 46 \\
\hline $\begin{array}{l}4 \text { Varactor stack } \\
\text { configuration [6] }\end{array}$ & 1.95 & - & $>0.2$ & OIP3 60 \\
\hline Single MEMS [7] & 10 & 40 & $\begin{array}{l}3 \\
(\max )\end{array}$ & IIP3 80 \\
\hline
\end{tabular}

Varactor diodes provide an excellent means of continuously tuning frequency but require high voltage bias 
lines to operate and are inherently non-linear in behaviour, where low power handling is an issue for single varactors. Low power handling is being addressed by incorporating multiple varactors in stacks or incorporating additional passive components [4], however this increases device count and area and may create additional complexities in terms of design and behaviour when components are placed in parallel configurations and signal is split between different paths.

PIN diodes are a more popular choice in reconfigurable circuits as they require bias lines with lower voltage, however they display higher loss due to their $\mathrm{ON}$ state resistance, compromising performance. A PIN diode used in series for a tunable filter has a reported IIP3 of $46 \mathrm{dBm}$ and distortion free output tested up to $2 \mathrm{~W}$ [5]. Non-linearity of PIN and varactor diodes has been acknowledged as a limiting factor in their use in high power reconfigurable circuits and alternative topologies are widely being researched to combat this problem. Results show linearity as a figure of OIP3 (Output Intermodulation Product) to be in the region of $60 \mathrm{dBm}$ when a collection of varactor diodes and passives are used in certain configurations [6], however single switches are far less linear. The Output Intermodulation Product is reported in this instance as opposed to IIP3 as a significant part of the input power is reflected by the varactor shunt impedance in this configuration. Since RF power is dissipated in the form of heat in the junction resistance of these devices, they have a lower mean time to failure [8] compared to junctionless switches.

MEMS switches have excellent insertion loss and isolation values and display far more linear behaviour, particularly as an alternative to varactor diodes; however they often display problems such as self actuation when handling higher input powers, particularly when hot-switched, reported to be $\sim 3 \mathrm{~W}$ [9], limiting their reliability. They are also more difficult to integrate into reconfigurable circuits, requiring high voltage biasing lines.

Photoconductive switches are seen as an attractive option as there is high isolation between the controlling optical beam and the RF input signal. In the application of reconfigurable antennas this may allow improved radiation pattern by eliminating interference from metallic biasing lines used to control circuit or antenna adaptability hence their removal has clear advantages. In addition to this, due to the absence of a junction in a photoconductive silicon die switch, the voltage at which the switch experiences breakdown is considerably higher, and hence there is potential for greater power handling capability.

Currently, a heterostructure light activated microwave photoconductive switch design [1] with flip chip light source has a reported third order intercept (TOI) of $65 \mathrm{dBm}$, extrapolated from measurements taken up to $1 \mathrm{~W}$. The heterostructure switch significantly differs from the silicon switch presented in this research as it comprises a complex 13 epilayer structure, involving a much more intricate set of manufacture processes. At its current state of development it is difficult to estimate production cost of the optically triggered switch, so price comparison is not made in this paper. Applications for which the switch would be ideal include long range high power transmitting antenna where minimum signal distortion is an important factor.

\section{SWITCH DESIGN}

The photoconductive switches consist of a die of phosphorus doped silicon, resistivity $>6000 \Omega$, attached between two lengths of copper microstrip line, Fig. 1. The die, measuring $1 \mathrm{~mm} \times 2 \mathrm{~mm} \times 0.3 \mathrm{~mm}$, is attached over the gap using silver epoxy. When illuminated by light of a wavelength of $\sim 980 \mathrm{~nm}$, electrons in the silicon become promoted from the valence band to the conduction band and electron-hole pairs are created hence making the silicon conductive. The switch is in its closed state when illuminated which allows RF signal to propagate through the silicon.

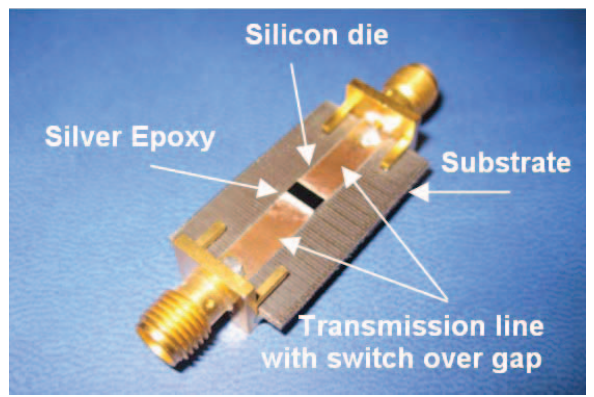

Fig. 1 Optically reconfigurable microstrip with a silicon switch

The illumination source is located remotely in the form of a laser diode and is delivered via a fibre optic cable. Once the light is removed the switch returns to its open state, behaving as an insulator and blocking RF signal through the silicon, the switch does not incur any degradation through this process. The gap width between the transmission line segments is $0.25 \mathrm{~mm}$.

\section{MEASUREMENT PLATFORM}

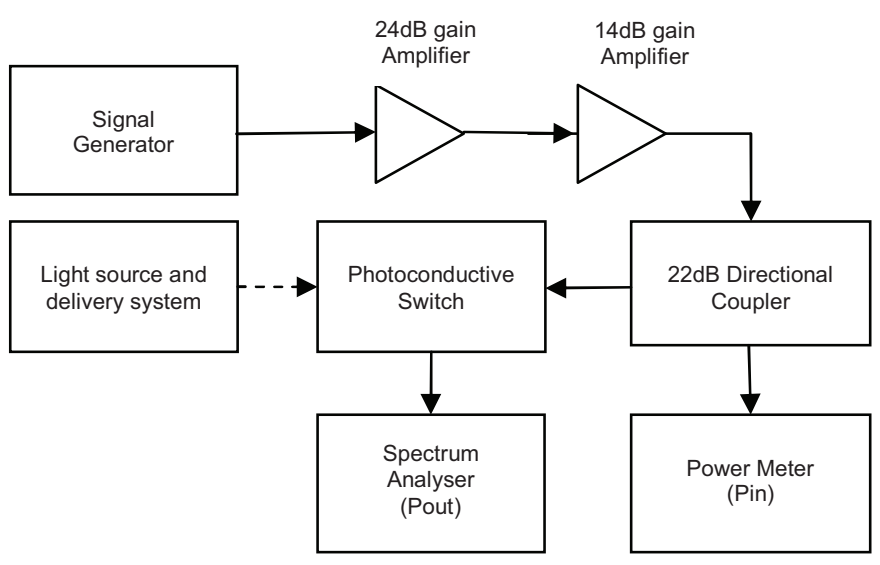

Fig. 2 Schematic of experimental test setup

\section{A. Experimental setup}

Parameters including insertion loss, isolation measurements and second and third order intercept (SOI/TOI) measurements are determined using the measurement platform illustrated in 
Fig. 2. An Anritsu 37397D Vector Network Analyser measures the S-parameter values to determine feed-through response in both on and off switch states. For the harmonic testing, signal is generated using a signal generator and is boosted to produce $1 \mathrm{~W}$ by external amplification using a combination of amplifier modules, Fig. 3.

A high directivity $22 \mathrm{~dB}$ directional coupler adapted from a design by Baek et al. [10] is used to couple a portion of the input signal entering the switch to determine an accurate power reading just before the switch. The output signal is fed into the Spectrum Analyser, Advantest R3132 to measure power at the fundamental frequency $\mathrm{f}_{0}, 2 \mathrm{GHz}$, and second and third harmonics at $2 \mathrm{f}_{0}(4 \mathrm{GHz})$ and $3 \mathrm{f}_{0}(6 \mathrm{GHz})$.

\section{B. Conditions and Limitations}

To determine the effect of optical power on switch linearity, optical power is varied between $10 \mathrm{~mW}$ and $200 \mathrm{~mW}$ and applied for 5 minutes before taking measurements so as to limit the effects of laser source optical power fluctuations. Insertion loss measurements are taken first to ensure optimum positioning of the fibre optic cable followed by power in/out measurements at the fundamental and second and third order harmonic readings. To ensure accuracy, harmonic measurements are taken from the signal generator and both power amplifiers to eliminate their effects on results.

All measurements are taken at ambient temperature in a fan ventilated room, $\sim 23^{\circ} \mathrm{C}$, with the switch located inside a laser safe structure. The third order intercept point is independent of frequency as well as capacitance value and so is suitable measure of linearity. An absolute measurement of power handling is the $1 \mathrm{~dB}$ compression point, however a suitable high power RF source was not available to take measurements up to such a high power value at this stage of the study but will be considered in future work.

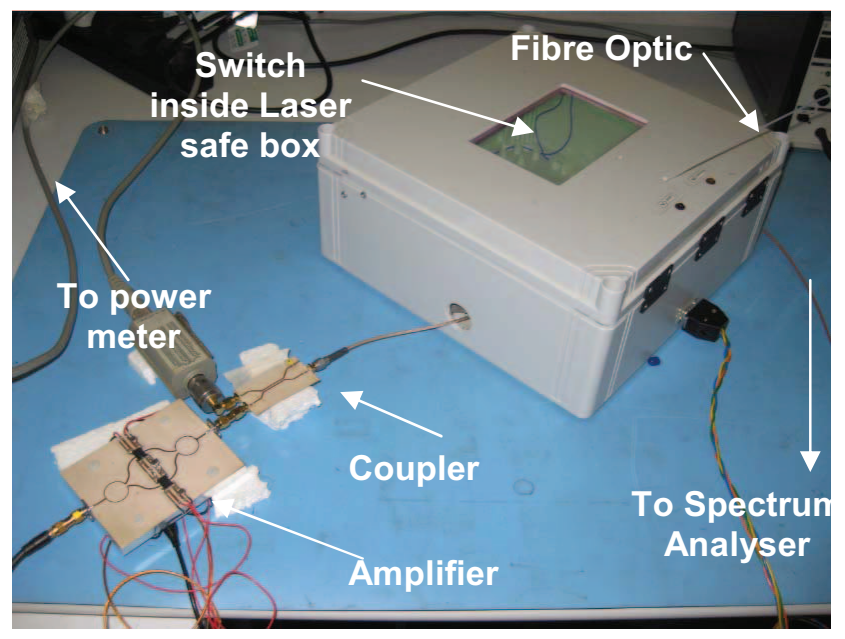

Fig. 3 Measurement platform for performing linearity testing on a silicon switch

\section{MEASUREMENTS AND DISCUSSION}

\section{A. Isolation and Insertion Loss}

Isolation results in the switch OFF state are presented in Fig. 4 along with $\mathrm{S}_{11}$. The isolation in the off state is measured at $15.5 \mathrm{~dB}$ at $2 \mathrm{GHz}$. The reflected signal, $\mathrm{S}_{11}$ in the off state is $0.19 \mathrm{~dB}$. The insertion loss whilst controlled by $200 \mathrm{~mW}$ of optical power is $0.69 \mathrm{~dB}$. Only a small amount of power is dissipated, hence system efficiency is not compromised by the switch. As minimum power is dissipated in the switch, there is also reduced thermal contribution that could potentially raise the temperature of the wireless system. $\mathrm{S}_{11}$ of the switch in the $\mathrm{ON}$ state is $-20.2 \mathrm{~dB}$. As optical power decreases, insertion loss increases, Fig. 5. At the minimum optical power of $10 \mathrm{~mW}$, insertion loss is $3 \mathrm{~dB}$, this supports work conducted by Panagamuwa et al. [2].

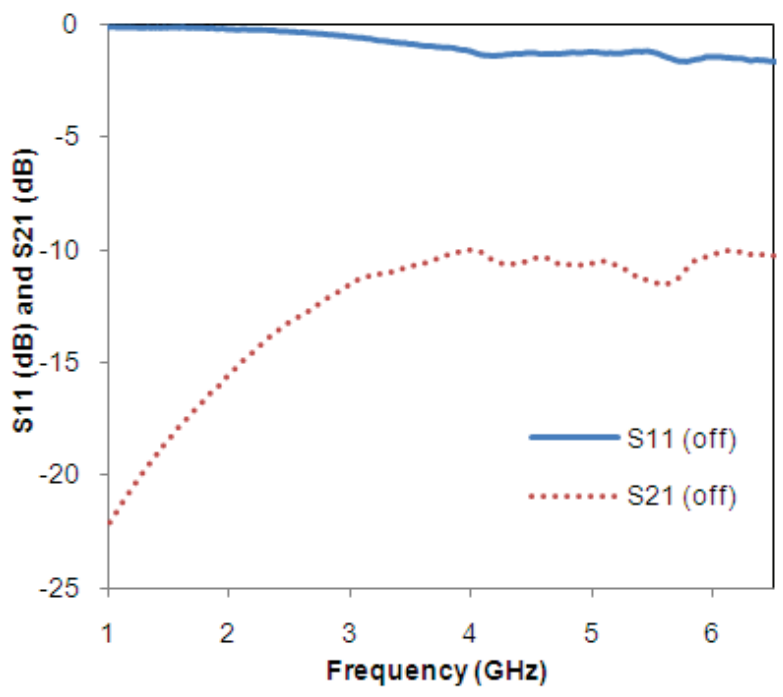

Fig.4 Off state S21 and S11 of the silicon switch

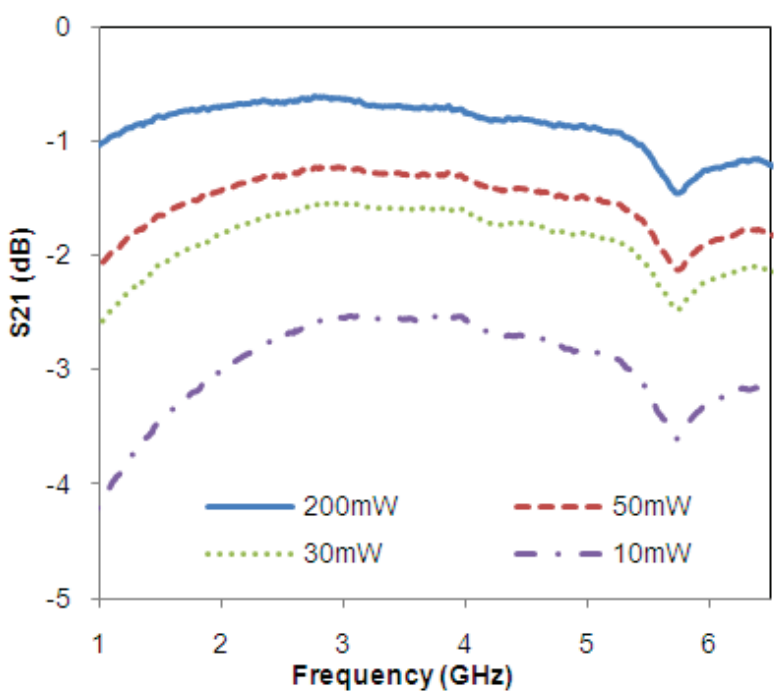

Fig 5 On state S21 under various optical control powers

\section{B. Linearity}

The fundamental output power versus input power is presented in Fig. 6. The switch remains linear over the full range of measurements and beyond $1 \mathrm{~W}$. An indication of linearity can be expressed as the SOI and TOI figures. Second and third order harmonics are also displayed and have been extrapolated to provide the second and third intercept points, $63 \mathrm{dBm}$ and $70 \mathrm{dBm}$ respectively referenced to the switch's 
output power. These measurements were taken with the switch under illumination of $200 \mathrm{~mW}$, note that the insertion loss of the switch at the second harmonic frequency $(4 \mathrm{GHz})$ is $0.75 \mathrm{~dB}$ and the corresponding insertion loss at the third harmonic frequency $(6 \mathrm{GHz})$ is $1.25 \mathrm{~dB}$, Fig. 5 .

Fig. 7 presents varying TOI with respect to optical control power. It can be observed that as illumination increases, linearity remains constant presenting the switch as a reliable mechanism for handling high power under any light intensity above $10 \mathrm{~mW}$. Linearity is independent of the control method. This contrasts with other microwave switches where device resistance is dependent on current supplied to the switch and can severely compromise linearity if the switch is not biased at the right level.

\section{Maximum Power Handling}

By means of visual inspection, there were no signs of distress to either the silicon die or silver epoxy bonding agent.

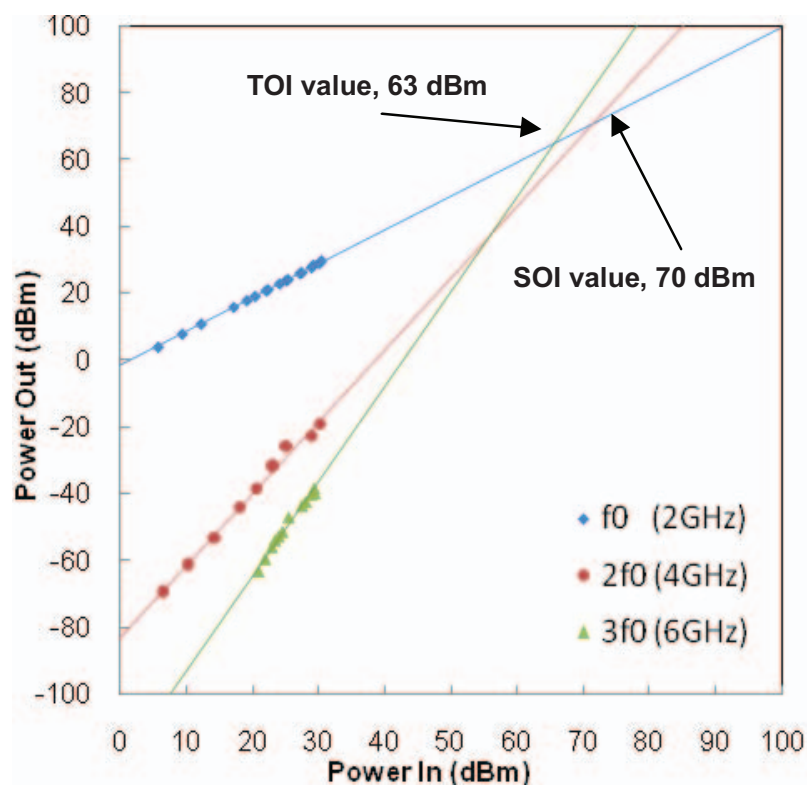

Fig.6 Fundamental linearity and second order and third order harmonics of switch under $200 \mathrm{~mW}$ of optical power, highlighting intercept points

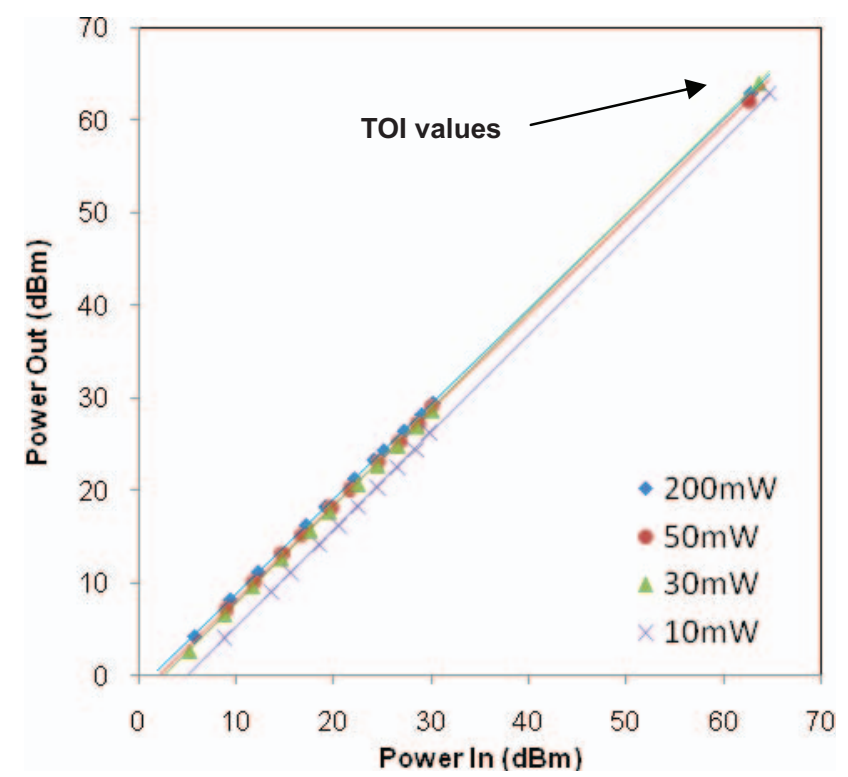

Fig. 7 Fundamental linearity under varying optical control powers including third order intercept points
The silicon switch does not appear to have been degraded by the RF input power, and performance remained constant up to the maximum test power of $1 \mathrm{~W}$.

\section{CONCLUSION}

The linearity of an optically controlled silicon switch has been presented in the form of second and third order intercept points. Characterization reveals an SOI value of $70 \mathrm{dBm}$ and TOI value of $63 \mathrm{dBm}$ - paving the way for photoconductive switches in a wide variety of high power RF applications. Switch linearity remained constant irrespective of optical intensity of the controlling light source.

To complement these projected linearity measurements, microwave performance of the optically activated photoconductive switch has also been reported. Isolation in the OFF state is $15.5 \mathrm{~dB}$, where $\mathrm{S}_{11}$ is $-0.19 \mathrm{~dB}$ at $2 \mathrm{GHz}$. Insertion loss in the $\mathrm{ON}$ state under $200 \mathrm{~mW}$ of optical power is $0.69 \mathrm{~dB}$, with a corresponding $\mathrm{S}_{11}$ of $-20.2 \mathrm{~dB}$. These results indicate that the anticipated power handling performance of any microwave system need not be compromised by the photoconductive switching element.

\section{FUTURE WORK}

Future work will involve fully characterizing the linearity of a photoconductive switch. This will include ascertaining the $1 \mathrm{~dB}$ compression point of the switch by measuring the device up to $30 \mathrm{~W}$, determining a true upper limit to switch linearity.

\section{REFERENCES}

[1] Y. Kaneko, T. Takenaka, T.S. Low, Y. Kondoh, D.E. Mars, D. Cook and M. Saito, "Microwave switch: LAMPS (light activated microwave photoconductive switch)," IEEE Electronic Letters, Jun 2003.

[2] C. J. Panagamuwa, A. Chauraya and J. C. Vardaxoglou, "Frequency and beam reconfigurable antenna using photoconducting switches" IEEE Trans. Antennas Propagat., vol. 54, no.2, pp. 449-454, 2006.

[3] D. Draskovic, C. Panagamuwa, J. C. Vardaxoglou, D. Budimir, "Frequency reconfigurable RF circuits using photoconducting switches," International Journal of RF and Microwave Computer-Aided Engineering, Vol. 20, No. 1, January 2010

[4] K. Buisman, C. Huang, A. Akhnoukh, M. Marchetti, L. C. N de Vreede, L. E. Larson and L. K. Nanver, "Varactor topologies for RF adaptivity with improved power handling and linearity," , IEEE/MTT-S International Microwave Symposium, pp. 319 - 322 , June 2007.

[5] C. Lugo Jr. and J. Papapolymerou, "Electronic switchable bandpass filter using PIN diodes for wireless Iow cost system-on-a-package applications," IEEE Proceedings Microwave Antennas Propagation, Vol. 151, No. 6, December 2004

[6] C. Huang et al., "Ultra linear low-loss varactor diode configurations for adaptive RF systems," IEEE transactions on microwave theory and techniques, Vol. 57, No. 1, January 2009.

[7] J. DeNatale, "RF MEMS Circuits: Applications of MEMS switch and tunable capacitor," Rockwell Scientific, June 2004, available online http://www.ibersensor.org, last accessed June 2010

[8] C. L Lim, "PIN diode switches for high-linearity applications," in EE Times-India, November 2008.

[9] E. R. Brown, "RF-MEMS switches for reconfigurable integrated circuits," IEEE Transactions on microwave theroy and techniques, Vol. 46, No. 11, November 1998

[10] D. Baek and Y. Kim, "Symmetric Microstrip interdigital capacitorcompensated high directivity directional-coupler," Microwave and optical technology letters, Vol 50, No 11, November 2008 